\title{
Influence of the Filling Oil Compounds and Additives on the Thermal Stability of Insoluble Sulfur
}

\author{
Wang Yan zhen ${ }^{1 *}$, Duan Hong ling1, Song Caixia ${ }^{2}$, Ma Yajuan ${ }^{1}$ and Song Chunmin ${ }^{1}$ \\ ${ }^{1}$ State Key Laboratory of Heavy Oil Processing, China University of Petroleum, China \\ ${ }^{2}$ Shenhua Ningxia Coal Group Coal Chemical Branch, China \\ *Corresponding author : Wang Yan Zhen, State Key Laboratory of Heavy Oil Processing, China University of Petroleum, Qingdao, Shandong, China. \\ Submission: 洞 March 19, 2018; Published: 眥 April 05, 2018
}

\begin{abstract}
The thermal stability is one of the most important properties of insoluble sulfur (IS). The filling oil directly influences the thermal stability of IS. The effect of basic organic nitrogen compounds and ash less stabilizers used in filling oil were studied to improve the thermal stability. The data showed that the thermal stability decreased with an increase in the basic nitrogen compound content in the filling oils. When the basic nitrogen content in the filling oil was lower than $20 \mu \mathrm{g} / \mathrm{g}$, the thermal stability of IS was not changed. Ash less stabilizers including phenols, esters, carboxylic acids and their mixtures could increase the thermal stability of IS. When different types of phenols were mixed, the thermal stability of IS was greater than $91 \%$. The thermal stability of IS reached $95.54 \%$ when $0.2 \mathrm{wt} \%$ of oleic acid was used.
\end{abstract}

Keywords: Insoluble sulfur; Basic nitrogen compounds; Thermal stability; Ash less stabilizer

\section{Introduction}

Insoluble sulfur (IS) is a type of sulfur polymer, which is insoluble in $\mathrm{CS}_{2}$ and is a good rubber vulcanizing agent [1-4]. There are two types of IS, oil-filled and non-oil-filled. Because non-oilfilled IS is a powder, it is not convenient for use and transport. In contrast, oil-filled IS can be dispersed evenly and be widely used. Therefore, IS should be treated with oil $[5,6]$. The production of IS usually adopts a high temperature method, which causes the liquid or gas sulfur to spray into cold liquids to obtain IS with a concentration of 30-60wt\%. To obtain high concentrations of IS, solvent has been used to extract the soluble sulfur, followed by filling with oil to obtain oil-filled IS [2,7-11].

The sulfur atom contains 6 electrons in its outer orbital, which is an unstable structure. Sulfur atoms in the middle of a polymer chain are stable because they can possess two covalent bonds with the adjacent sulfur atoms, resulting in 8 electrons in the outer orbital, which is a stable structure. However, the two atoms at the ends of the polymer chain possess only 7 electrons, which is an unstable structure. If the IS is not treated with a stabilizer, the sulfur chain may break due to the two ends and become soluble sulfur. Therefore, stabilizers are used in the process of IS production to inhibit breakage of the sulfur chain [12-16]. The selection and use of stabilizers is crucial to IS production. The stabilizers can be used in the cooling liquid to increase the yield and stability of IS. Metal salts are now used; however, they can add to the ash content of IS and may have negative effects on the rubber properties. Ashes less stabilizers are very important for IS. The stabilizers are also used in the filling oil. The filling oil is an important factor for the IS stability, and their components influence the stability and dispersion of IS $[17,18]$. There are few studies on the influence of the components and ash less stabilizers in the filling oil. The aim of this paper is to study these effects.

\section{Experimental}

\section{Materials and reagents}

Crude IS, which was produced by a sulfur gas method, was sampled from a factory in China. $\mathrm{CS}_{2}$, oleic acid, salicylic acid, turpentine, triphenylphosphate, triphenylphosphite, and dodecanoic acid were chemically pure reagents. 2,6-Di-tert-butyl4-methylphenol (reagent A), 4,4'-thio-bis(6-tert-butyl-m-methyl phenol) (reagent B), and pentaerythritoltetrakis 3-(3,5-ditertbutyl-4-hydroxyphenyl) propionate (reagent $\mathrm{C}$ ) were greater than 99\% pure. Filling oil was sampled from an oil refinery in China. A type of nitrogen removal reagent was sampled from Dongying Hetai Petroleum Science \& Technology Corp. LMT. Activated clay powder was sampled from a lubricating oil producer.

\section{Extraction of crude IS}

The crude IS (approximately $100 \mathrm{~g}$ ) was extracted twice using $200 \mathrm{ml}$ of $\mathrm{CS}_{2}$ at room temperature for $30 \mathrm{~min}$, and then the IS was filtered and dried at $60^{\circ} \mathrm{C}$ for $1 \mathrm{~h}$ to obtain pure IS with a concentration greater than $90 \mathrm{wt} \%$. 


\section{Oil filling}

Extracted IS $(10 \mathrm{~g}), 110 \mathrm{~g}$ of filling oil and $0.1-1 \mathrm{~g}$ of stabilizer were charged into a $500 \mathrm{ml}$ three-neck flask. The mixture was heated to $70{ }^{\circ} \mathrm{C}$ and was stirred for $30 \mathrm{~min}$. Then, the mixture was vacuum filtered to remove the excess oils.

\section{Removal of basic nitrogen compounds from the filling oils}

Approximately $300 \mathrm{~g}$ of filling oil and $6 \mathrm{~g}$ of nitrogen removal reagent were charged into a $500 \mathrm{ml}$ three-neck flask with a temperature controller and an electric stirrer. The temperature was controlled at $80^{\circ} \mathrm{C}$, and the reaction time was $20 \mathrm{~min}$. Then, the flask was placed into an electrically heated drying cabinet for $2 \mathrm{~h}$ at 80 ${ }^{\circ} \mathrm{C}$. The oil was separated into two parts. The complexes composed of the nitrogen removal reagent and the basic nitrogen compounds deposited in the bottom of the flask. The upper oil component was poured into another flask, and 3-9g of activated clay was charged into the new flask. The mixture was stirred, and the temperature was controlled at $180^{\circ} \mathrm{C}$ for $30 \mathrm{~min}$ under flowing $\mathrm{N} 2$ to protect the oil from oxidation. Then, the oil was filtered to remove the clay.

\section{Determination of the oil content in the IS}

The oil content was determined by solvent extraction. Oil filled IS $(10 \mathrm{~g})$ and $30 \mathrm{ml}$ of $\mathrm{n}$-hexane were charged into a $100 \mathrm{ml}$ flask, and the mixture was stirred for $20 \mathrm{~min}$. Then, the mixture was filtered. The remained IS was treated again according to the above method. The filtrates were vaporized and dried at $110^{\circ} \mathrm{C}$ for $2 \mathrm{~h}$ under -0.07 MPa to remove the solvent. The oil content of the IS was determined as the percentage of the recovered oil weight versus the IS weight.

\section{Determination of the thermal stability of the IS}

A metal test tube with a length of $150 \mathrm{~mm}$ and a diameter of $30 \mathrm{~mm}$ was pre-heated in glycerol at $105^{\circ} \mathrm{C}$ for more than $10 \mathrm{~min}$. IS $(5 \mathrm{~g})$ was charged into the test tube for $15 \mathrm{~min}$ at $105^{\circ} \mathrm{C}$, and then the test tube was removed and immediately cooled in cold water. $\mathrm{CS}_{2}(30 \mathrm{ml})$ was charged into the test tube and stirred for $10 \mathrm{~min}$ to dissolve the soluble sulfur. The solution was then filtered. Next, the IS was extracted and filtered again with $30 \mathrm{ml}$ of CS2 to remove all of the soluble sulfur. The remaining IS was dried in a drier at $60^{\circ} \mathrm{C}$ for $2 \mathrm{~h}$. The thermal stability was calculated according to Equation (1).

$$
\text { Thermal stability }=\frac{\text { IS weight after extraction }}{\text { IS weight-oil weight in IS }} \times 100 \%
$$

\section{Results and Discussion}

\section{Effect of basic nitrogen compounds in the filling oil}

The basic nitrogen compounds in the filling oil were removed by the nitrogen removalreagent. The nitrogen-removed oil and the untreated oil were mixed to obtain oils with different basic nitrogen contents. The effect of basic nitrogen compounds on the thermal stability is shown in Figure 1.

Figure 1 shows that the thermal stability of IS decreases with an increase in the basic nitrogen content in the filling oils. When the basic nitrogen content is lower than $10 \mu \mathrm{g} / \mathrm{g}$, it has a small effect on the thermal stability of IS. Therefore, the basic nitrogen content in filling oils should be lower than $10 \mu \mathrm{g} / \mathrm{g}$. The basic nitrogen compounds in oils are mainly organic amines, pyridines and quinolones. The lone pair electrons on the nitrogen atoms of the basic nitrogen compounds can induce the electrons of the IS chain and lessen the strength between the sulfur atoms. In addition, the nitrogen atoms may complex with the stabilizers of the outer layer sulfur atoms. These effects can result in the collapse of the polymer chain.

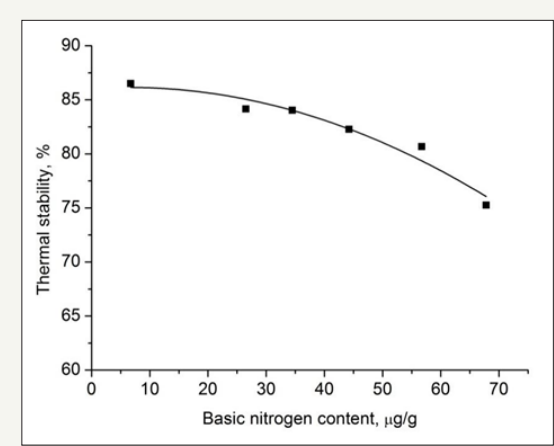

Figure 1: Effect of oils with different basic nitrogen contents on the thermal stability of IS.

\section{Effect of phenols}

2, 6-Di-tert-butyl-4-methylphenol (reagent A), 4, 4'-thio-bis (6-tert-butyl-m-methyl phenol) (reagent B) and pentaerythritoltetrakis 3-(3, 5-ditert-butyl-4-hydroxyphenyl) propionate (reagent $\mathrm{C}$ ) are usually used as antioxidants in rubbers. The effects of A, B and C are shown in Figure 2.

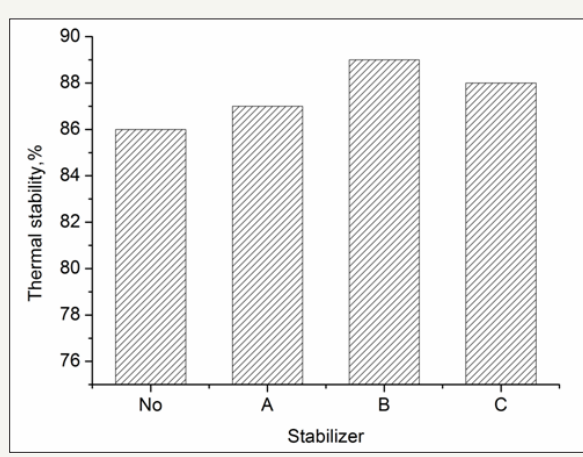

Figure 2: Influence of different stabilizers on the thermal stability of IS with nitrogen removal and oil treatment.

Figure 2 shows that reagents $\mathrm{A}, \mathrm{B}$ and $\mathrm{C}$ are beneficial to the IS stability. Reagent $\mathrm{B}$ is the best phenol stabilizer and has the best thermal stability (89.0\%). The phenol can form a negative phenoxy ion, which can couple with the free radicals on the two ends of the sulfur chain. Easier production of the negative phenoxy ion results in a stronger coupling strength and a higher thermal stability. The phenol structure influences the production of negative phenoxy ions. The hydroxyl groups in the phenols are linked with the carbon atoms of the aromatic ring. The conjugating effect of the hydroxyl groups and the aromatic ring makes it easy to delocalize the electrons of the hydroxyl groups, which frees the $\mathrm{H}+$ and produces negative phenoxy ions. The substituents may influence the stability 
of the negative phenoxy ions. The electron-donating groups in the aromatic ring may cause the phenoxy negative ions to be unstable, whereas the electron-withdrawing groups have an opposite effect.

\section{Effect of carboxylic acids}

Carboxylic acids can provide the coupling electrons for the outer layer atoms of the IS chain. The effects of lauric acid and oleic acid are shown in Figure $3 \& 4$

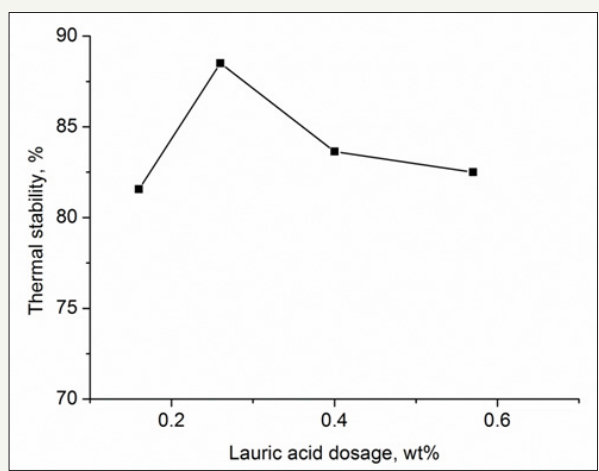

Figure 3: Effect of lauric acid.

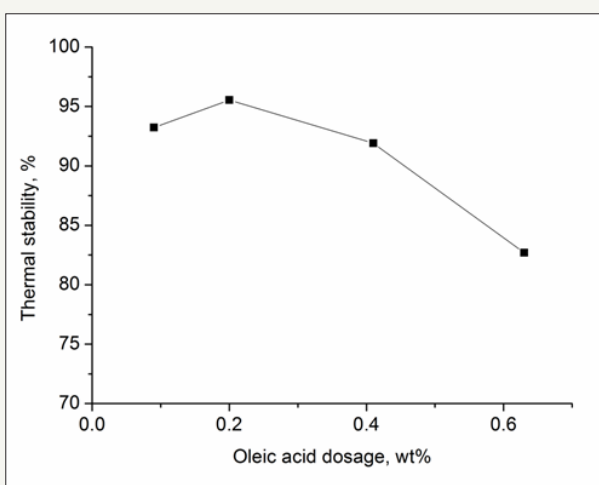

Figure 4: Effect of oleic acid.

Figure $3 \& 4$ show that lauric acid and oleic acid are both favorable for IS stability When the dosage of oleic acid was $0.2 \mathrm{wt} \%$ of the IS, the thermal stability was greater than $95 \%$. In addition, the stability may decrease when the stabilizer dosage is not in the optimal range. The carboxylic acids can provide the ion -COO-, which is an electron-donating group. The coupling of ion -COOand the lone pair electrons result in increased IS stability. However, when the carboxylic acid dosage is excessive, the excess -COO- ions can couple with the middle sulfur atoms and cause the collapse of the polymer chain. Oleic acid has a greater effect than lauric acid possibly due to the joint effects of the double bonds in oleic acid and the -COO- ion.

\section{Effect of turpentine oil}

Turpentine oil contains double bonds that may improve the thermal stability of IS. The effect of turpentine oil is shown in Figure 5.

Figure 5 shows that the IS stability can reach $91.5 \%$ when the turpine oil dosage is $0.5 \%$. Increasing or decreasing the dosage may lessen the effect. Turpentine oil contains pinenes, which consist of many aromatic rings. In addition, the electron density is greater than a benzene ring. Therefore, the stabilizing effect is greater than single ring molecule.

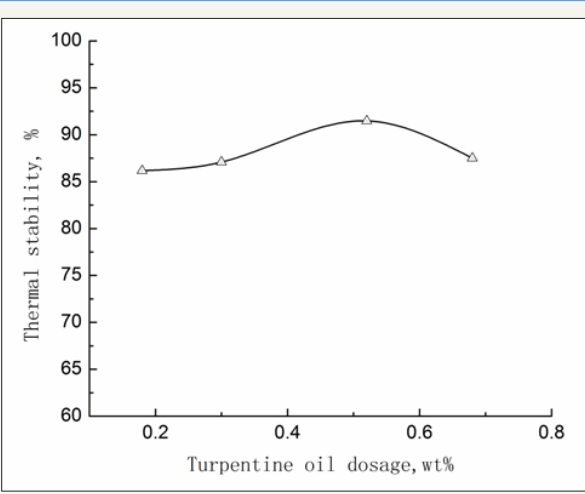

Figure 5: Effect of turpentine oil.

\section{Effect of phosphates}

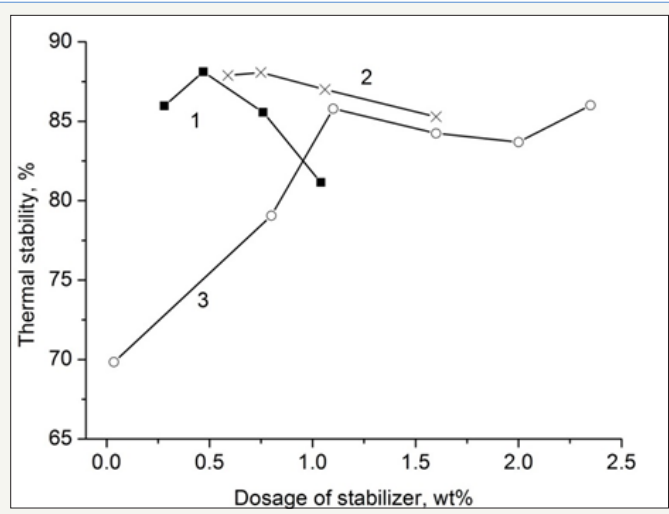

Figure 6: Effect of phosphates (1. triphenylphosphite in denitrogenated oil; 2. triphenyl phosphate in denitrogenated oil; 3. triphenyl phosphate in un-denitrogenated oil).

The effects of phosphates are shown in Figure 6. Figure 6 shows that the addition of phosphates is beneficial to the IS stability. The effect of phosphates is more evident when the basic nitrogen compounds in the filling oilsare removed, and the effect of the phosphates in the denitrogenated oil is better than that in the undenitrogenated oil. This is because the phosphates can contribute unoccupied orbital which may receive the electrons of the two ends of IS molecules.Further, triphenylphosphite and triphenyl phosphate display acidity which may complex with the basic nitrogen compounds to lower the side effect of the basic nitrogen compounds.

\section{Effect of mixed stabilizers}

The effects of mixed stabilizers are shown in Figure 7. The differentstabilizers are mixed according to the best dosage of individual stabilizers. Figure 6 shows that the effects of mixed stabilizers are better than the effects of individual stabilizers. The stability of the mixture containing phenol and phosphates was greater than $91 \%$. The synergistic effects of phenols and phosphates strengthen the structure of the sulfur polymer chain. 


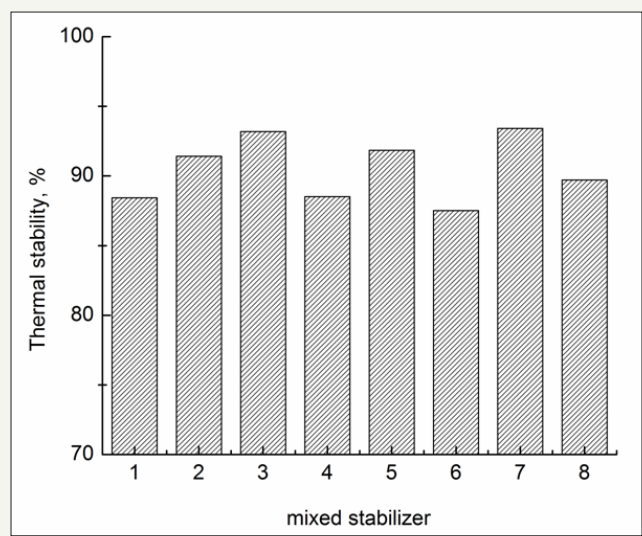

Figure 7: Effect of mixed stabilizers (1. C; 2. C+ triphenylphosphite; 3. C+triphenyl phosphate; 4. B; 5. B+triphenyl phosphate; 6. A; 7. A+triphenyl phosphate; 8. A+triphenylphosphite).

\section{Conclusion}

The component of filling oil is important to the thermal stability of insoluble sulfur. Basic nitrogen compounds clearly influence the IS stability. Low basic nitrogen content is beneficial for the IS stability. The basic nitrogen content should be lower than $10 \mu \mathrm{g} / \mathrm{g}$. The phenols, carboxyl acids and phosphates in filling oils can increase the thermal stability of IS. The effect of a mixture of a phenol and a phosphate in the filling oil is better than the individual effect.

\section{References}

1. Kozak, Zdzislaw (1978) Elementary sulphur insoluble in carbon disulfide.

2. Yuan H, Wang Y, Liu C (2006) Manufacture of IS by gasification method. Rubber Industry 53(9): 550-552.

3. Qiu Z, Chen Y, Zhang Y (2002) Study on the purification and hightemperature stability of insoluble sulfur. Journal of Nanchang University 26(2): 103-105

4. Han S, Liu C, Shi K (2002) The stabilization of high qualitative insoluble sulfur with halogen stabilizer. Journal of Taiyuan University of Technology 33(1): 26-29.

5. Fuchs H (1987) Vulcanizable rubber compounds containing insoluble sulfur.

6. Yuan H, Wang Y, Liu C (2006) Modified method for the manufacture of an oil treated insoluble sulphur. Specialty petrochemicals 23(6): 13-16.
7. Borokhovakii VA, Gordienko EG, Datsko RP (1982) Insoluble sulfur.

8. Deak M (1987) Manufacture of insoluble sulfur.

9. Reutskij VF (1981) Method of producing insoluble sulfur.

10. Shi K, Chen B, Liu C (1996) Investigation on new technology of insoluble sulphur production. Journal of Chemical Industry and Engineering 47(2): 254-258.

11. Ouyang FS, Bi Y, Li B, Sun Q, Weng H (2008) Effect of the molecular structures of extractants on separating insoluble sulfur. Journal of East China University of Science and Technology 34(4): 482-486.

12. Yukitatsu S (2004) Insoluble sulfur composition.

13. Wu WL, Wang Y, Zeng CY, Wang XH, Zhou LJ (2005) Influence of inorganic halides on stability of insoluble sulfur. Journal of Chemical Engineering of Chinese Universities 20(3): 356-361.

14. Rademacher T, Schober R (1965) Improvements in and relating to the production of electrostatically uncharged insoluble sulphur.

15. Macho, Vendelin (2000) Process for manufacture of insoluble sulfur or a mixure of soluble with insoluble sulfur and/or their mixture with hydrocarbon oil

16. Kyung SS, Irviogtom NY (1982) Process for the production of insoluble sulfur.

17. Yamanaka, Masami (1987) Sulfur composition containing insoluble sulfur.

18. Katayama T, Tamura N (1988) Process oil of insoluble sulfur for rubber.
Creative Commons Attribution 4.0 International License

For possible submissions Click Here

\section{Submit Article}

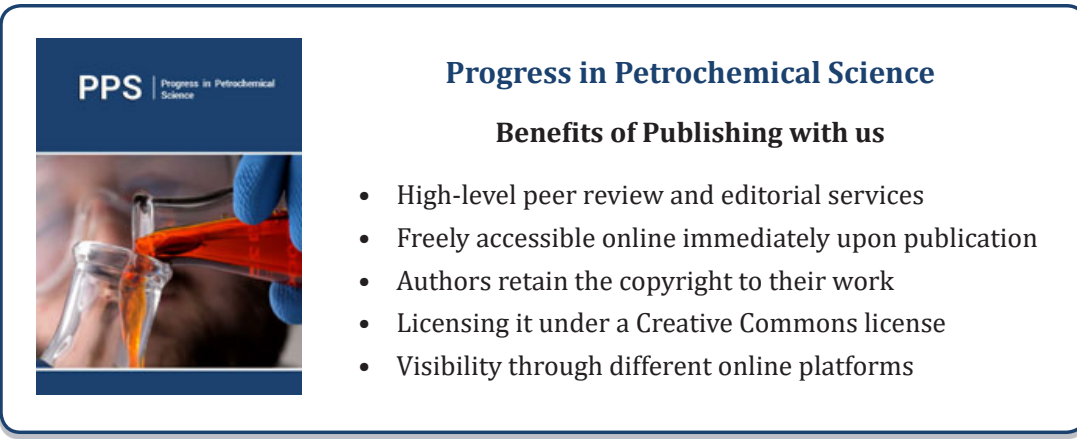

